\title{
Control of Energy Storage Systems for Aeronautic Applications
}

\author{
G. Canciello, A. Cavallo, and B. Guida \\ Department of Industrial and Information Engineering, University of Campania L. Vanvitelli, Aversa, Italy \\ Correspondence should be addressed to A. Cavallo; alberto.cavallo@unicampania.it
}

Received 13 April 2017; Accepted 8 June 2017; Published 19 July 2017

Academic Editor: Carlos-Andrés García

Copyright (C) 2017 G. Canciello et al. This is an open access article distributed under the Creative Commons Attribution License, which permits unrestricted use, distribution, and reproduction in any medium, provided the original work is properly cited.

\begin{abstract}
Future aircraft will make more and more use of automated electric power system management onboard. Different solutions are currently being explored, and in particular the use of a supercapacitor as an intelligent energy storage device is addressed in this paper. The main task of the supercapacitor is to protect the electric generator from abrupt power changes resulting from sudden insertion or disconnection of loads or from loads with regenerative power capabilities, like electromagnetic actuators. A controller based on high-gain concepts is designed to drive a DC/DC converter connecting the supercapacitor to the main electric bus. Formal stability proofs are given for the resulting nonlinear system, and strong robustness results from the use of high-gain and variable structure control implementation. Moreover, detailed simulations including switching devices and electrical parasitic elements are provided for different working scenarios, showing the effectiveness of the proposed solution.
\end{abstract}

\section{Introduction}

In current aircraft, energy is stored under the form of liquid hydrocarbon fuel by using Energy Storage Systems (ESS), which is burned with air in the engines. The ESS on operating commercial aircraft typically consists of fuel tanks, valves, sensors, and transfer pumps with piping, to move fuel to the engines [1]. In recent years, thanks to the More Electric Aircraft [2] (MEA) investigations, electrification of aircrafts started, aiming at completely replacing kerosene with electricity as primary energy source, within a not-so-far future. However, still many technical limitations exist before reaching this ambitious objective. One of these is related to the intelligent management of the electrical energy [3] accumulated in ESS such as batteries, fuel cells, supercapacitors, and other types of ESS, aiming at an overall reduction of weight carried onboard. In order to minimize the total amount of energy required for a full mission flight time, there is a stringent need for passing from static to dynamic management of electrical energy accumulated in ESS [4]. Currently, batteries and other ESS are providing energy only during particular flight phases (e.g., at the auxiliary power unit start-up or in emergency conditions). By allowing the electrical energy from the ESS to be reused also in different scenarios, such as for coping with temporary overloads of the generators [5] or for storing the energy regenerated by Electromechanical Actuators (EMAs) [6], significant savings can be obtained in terms of electrical machine and wiring weights reduction, with consequent minimization of the aircraft overall energy burns. Key elements in order to obtain a proper management of the electrical energy stored by ESS are bidirectional DC/DC converters [7], able to manage power flows in Buck mode (i.e., recharging the ESS) and in Boost mode (i.e., moving energy from the ESS to a voltage bus). For the scopes of an electricity intelligent management [8], traditional PI control strategies aiming at just maintaining the ESS recharge/discharge set points, both in voltage and in current, are no longer effective. Thus, fast and robust controllers shall be developed in order to ask ESS DC/DC converters to dynamically variate their operating modes and references. Moreover, such controllers shall be able to communicate in real-time with a supervisory control [9], in charge of monitoring the electrical network parameters (e.g., the generator power) and deciding the set points. In this paper, a high-gain control based on sliding manifold approaches $[10,11]$ is proposed for controlling the operations of a bidirectional DC/DC converter, connected to a supercapacitor-based ESS. The basic aeronautical electrical network here considered comprises two DC busses onboard, a high voltage (HV) bus, supplied by an electric generator 
followed by a voltage rectifier, and a low voltage (LV) bus, that is supplied by a supercapacitor-based ESS. Having this configuration, the energy management objective is twofold. First, when loads are abruptly connected to the bus, the ESS can be employed for supplying a quick energy pulse to the load, so as to reduce peaks on the generator [12]. Different shapes for this pulse have been examined [13], but in synthesis an exponentially shaped pulse results in simple implementation and good performances. Second, by using ESS and the proposed control strategy, the EMA braking energy can be stored for successive controlled release, with the double benefit of deleting the heavy and bulky dissipation resistor typically introduced on the HV bus, moreover reducing the risk of electrical shocks to other systems (especially the generator). This idea has been presented in different context [2], but, to the best of our knowledge, a formal proof of stability and detailed simulations to confirm the effectiveness of the concept has not presented before. It has been simply supposed that the current in the supercap follows a prescribed reference, without details on the control policy used.

In this paper some ideas about the intelligent management of ESS devices are presented, along with formal proofs of stability of the proposed control laws. We will refer to a minimal configuration including a generator, one supercap and a variable load, as shown in Figure 1. The control strategy prescribes sharp profiles of energy the supercap has to deliver (or to absorb, depending on the load variation). Then the low-level controller, based on sliding manifold and highgain methodologies, activated the converter's switches so as to manage the required power peaks. The final implementation is obtained with switching devices, by using known mathematical properties of Variable Structure Systems [14]. Ancillary problems, like recharging the supercap based on the state-of-charge, have not been directly addressed but can be treated in the same way as the peaking management. Indeed, managing the state-of-charge can be done simply by defying a suitable reference current for the converter [15] that is a special case of the control problem addressed in this paper. Detailed simulations are presented with switching components in MATLAB/Power System Blockset, considering different operating scenarios. Comparison with scenarios without supercap shows the effectiveness of the proposed strategy.

\section{System Model}

The system in Figure 1 is composed by a ESD, a variable load and a HV generator. As stated in Introduction, the voltage on the HV side is produced by a controlled electric generator followed by an uncontrolled rectifier bridge and, for modelling purposes, it is represented by an ideal voltage source $E_{H}$ and its internal resistance $R_{H}$. In order to model active power requested by a load on the $\mathrm{HV}$ side, a simple resistor $R_{D}$ is used. The ESD is connected to the HV bus through a DC/DC bidirectional converter. Thus, the converter is an integral part of the ES system. The schematic of the proposed configuration is shown in Figure 2, where a supercapacitor is used as an EDS.

The switches are operated in complementary mode; that is, $S_{1}$ is on when $S_{2}$ is off, and vice versa. The equations in

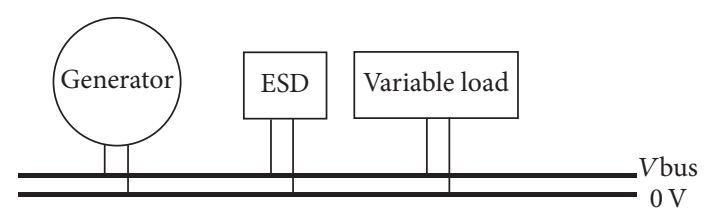

FIGURE 1: Basic energy management configuration.

both configurations ( $S_{1}$ on, $S_{2}$ off) and ( $S_{1}$ off, $S_{2}$ on) are easily derived and can be described in a compact way by using a binary control variable and the control $u \in\{0,1\}$ defining the two configurations. The resulting mathematical model is

$$
\begin{aligned}
& \dot{x}_{1}=-\frac{R_{\mathrm{ESR}}}{L} x_{1}+\frac{1}{L} x_{2}-\frac{1}{L} x_{3} u, \\
& \dot{x}_{2}=-\frac{1}{C_{\mathrm{SC}}} x_{1}-\frac{1}{R_{\mathrm{EPR}} C_{\mathrm{SC}}} x_{2}, \\
& \dot{x}_{3}=\frac{1}{C_{\text {bus }}} x_{1} u-\frac{1}{C_{\text {bus }}}\left(\frac{1}{R_{H}}+\frac{1}{R_{D}}\right) x_{3}+\frac{E_{H}}{R_{H} C_{\text {bus }}} .
\end{aligned}
$$

Here $x_{1}$ is the current flowing through the inductor $L, x_{2}$ is the voltage on the supercapacitor $C_{\mathrm{SC}}$, and $x_{3}$ is the voltage on the bus capacitor $C_{\text {bus }}$. Since the focus in this paper is on the current flowing in the supercap, the output $y$ is

$$
y=x_{1} \text {. }
$$

The aim of the control design is to define the switching sequence of the control $u$ so that the output $y$ follows a desired reference. This is the described in the next Section.

\section{Control Design}

The controller is designed by considering two logical operations. At high level, the desired output $\bar{y}(t)$ has to be selected for each operation phase of the system. Next, at low level, a controller has to be designed to solve a tracking problem, so that the inductor current $y$ can accurately follow the reference $\bar{y}(t)$. The two issues are separately described in the following paragraphs.

3.1. Definition of Current Reference. Note that the supercap acts only during transient phases; that is,

(i) when the current requested to the generator by the loads suddenly increases, the supercap supplies an extracurrent while the generator smoothly increases its current;

(ii) dually, when the current request abruptly decreases, the supercap absorbs the initial extracurrent from the generator, allowing the generator to gently reduce its current.

Moreover, the DC/DC converter interfacing the supercap to the $\mathrm{HV}$ bus can be assumed lossless, so that a simple power balance before and after the converter yields

$$
V_{\mathrm{SC}} x_{1}=x_{3} I_{\mathrm{ESD}}
$$




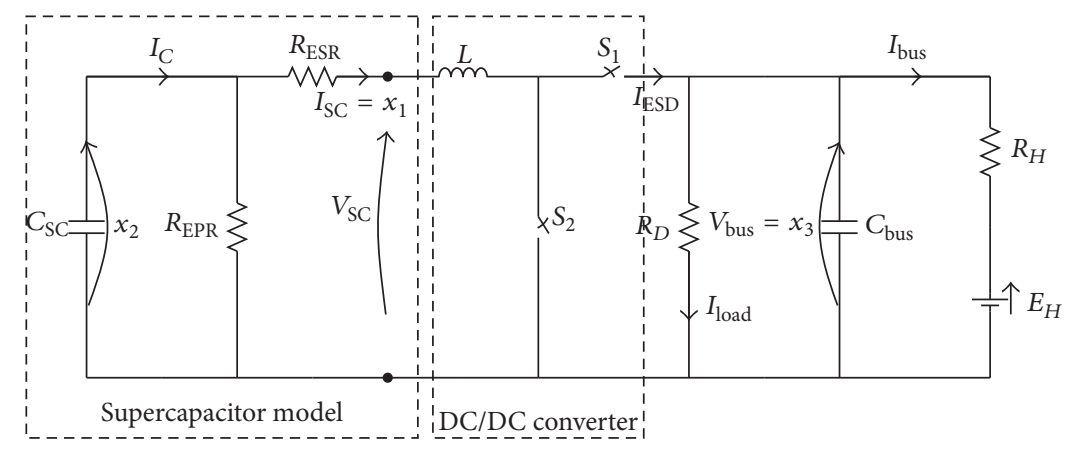

FIgURe 2: System.

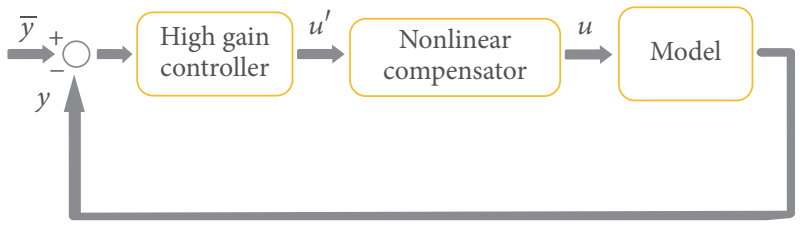

FIGURE 3: Control scheme.

Based on the above considerations, we can compute the desired current $x_{1}$ in the converter inductance. If we consider the worst case load current demand, that is, a sharp, stepwise shaped function, and a simple first-order exponential shape as an admissible generator current, a simple profile for the reference current $\bar{y}$ as the load changes is

$$
\bar{y}(t)=\frac{x_{3}(0) \Delta I_{\mathrm{ESD}}(0)}{V_{\mathrm{SC}}(0) e^{-\theta t}}=\bar{y}(0) e^{-\theta t}
$$

assuming that the load change happens at time $t=0$, where $1 / \theta>0$ is the time constant defining the duration of the supercap action and $\Delta I_{\mathrm{ESD}}(0)=I_{\mathrm{ESD}}\left(0^{+}\right)-I_{\mathrm{ESD}}\left(0^{-}\right)$.

Note that the sudden change in $I_{\mathrm{ESD}}$ is essentially caused by the current variation in the load. In fact, as the load changes, the current in the capacitor $C_{\text {bus }}$ has a small change (since the voltage $x_{3}$ is essentially unchanged) so we have $I_{\mathrm{ESD}} \simeq I_{\text {load }}+I_{\text {bus }}$. Since we want $\Delta I_{\text {bus }}(0) \simeq 0$ we must impose

$$
\Delta I_{\mathrm{ESD}}(0)=\Delta I_{\mathrm{load}}(0)
$$

3.2. Tracking Controller Design. System (1)-(3) has two nonlinear (actually, bilinear) equations and a linear one. The first equation can be linearized by using a simple variant of a nonlinear compensator proposed in [4]

$$
u=\frac{x_{2}-R_{\mathrm{ESR}} x_{1}-u^{\prime}}{x_{3}} .
$$

The nonlinear compensator manipulates the signal produced by the controller, as depicted in Figure 3.
In this way, (1)-(3) turn into

$$
\begin{aligned}
\dot{x}_{1}= & \frac{1}{L} u^{\prime}, \\
\dot{x}_{2}= & -\frac{1}{C_{\mathrm{SC}}} x_{1}-\frac{1}{R_{\mathrm{EPR}} C_{\mathrm{SC}}} x_{2}, \\
\dot{x}_{3}= & \frac{1}{C_{\mathrm{bus}}} x_{1} \frac{x_{2}-R_{\mathrm{ESR}} x_{1}-u^{\prime}}{x_{3}} \\
& -\frac{1}{C_{\text {bus }}}\left(\frac{1}{R_{H}}+\frac{1}{R_{D}}\right) x_{3}+\frac{E_{H}}{R_{H} C_{\mathrm{bus}}} .
\end{aligned}
$$

Note that system (9)-(11) is still nonlinear, but with only one nonlinear equation, making the control design simpler. Specifically, the tracking controller proposed in this paper is based on a high-gain sliding manifold control approach. The use of sliding control strategies has been widely addressed in the literature, especially due to its robustness properties.

For the sake of simplicity, we will still refer to a single load change at time $t=0$. Next the general case will be discussed. Define a sliding manifold as the set of points such that

$$
\mathcal{S}=\{(t, y) \mid \sigma(t, y)=0, \forall t \geq 0\},
$$

where the sliding function $\sigma$ is

$$
\sigma(t, y)=L\left[\bar{y}(t)-y-e^{-c t}(\bar{y}(0)-y(0))\right],
$$

where the constant $c>0$ is a free parameter. Note that $\sigma(0, y(0))=0$. From a practical point of view, the voltage $x_{3}$ across the HV capacitor is always positive (it would be zero in the case of infinitely many loads, so that $R_{D}=0$ ), and for this reason we will refer to the solution in the domain $\mathscr{D}=\left\{x \in \mathbb{R}^{3} \mid x_{3}>0\right\}$. Also from a physical point of view the assumption $x_{3}>0$ makes sense, since reversing the voltage on the HV capacitor means to reverse the flow of power.

The following theorem gives the conditions for designing a stabilizing controller to track the reference signal $\bar{x}_{1}(t)$.

Theorem 1. Consider system (9)-(11) in the domain $\mathscr{D}$ with the control law

$$
u^{\prime}(t)=\frac{1}{\epsilon}\left(\sigma\left(t, x_{1}\right)+\gamma \int_{0}^{t} \sigma\left(\tau, x_{1}\right) d \tau\right)
$$


where $\gamma$ is a positive constant and $\epsilon>0$ is a "small" constant, and let $x_{i}(t, \epsilon), i=1, \ldots, 3$, denote solution of the closed-loop system. Assume that $x(0) \in \mathscr{D}$. Then for any $\delta>0$, there exist $\epsilon^{*}>0, T=T(\epsilon)>0, C>0$, and $\Theta>0$ such that, for all $0<\epsilon \leq \epsilon^{*}, c>C, \theta>\Theta$, the closed-loop system is stable and such that

$$
\left|\bar{y}(t)-x_{1}(t, \epsilon)\right|<\delta, \quad \forall t>T .
$$

Proof. The control law (14) is the solution of the differential equation

$$
\epsilon \dot{u}^{\prime}=\dot{\sigma}+\gamma \sigma
$$

Now we use the theory of singular perturbations to system (9)-(11) and (16) with the small parameter $\epsilon$ and use Tikhonov's theorem [16]. The fast subsystem is stable due to (9), (13), and (16). In order to prove the stability of the slow subsystem, let $\epsilon=0$ and solve $\dot{\sigma}=0$ for $u^{\prime}$, obtaining the so-called equivalent control [17]

$$
u_{\mathrm{eq}}^{\prime}=-L\left(c(y(0)-\bar{y}(0)) e^{-c t}+\theta \bar{y}(0) e^{-\theta t}\right) \text {. }
$$

Note that the conditions $\dot{\sigma}=0$ and $\sigma(0, y(0))=0$ imply $\sigma(t, y(t))=0$ for all $t \geq 0$. Then $x_{1}$ is computed as

$$
x_{1}=\bar{y}(t)-e^{-c t}(\bar{y}(0)-y(0)) \equiv \varphi_{1}(t ; c, \theta),
$$

where $\varphi_{1}$ is a scalar function such that $\lim _{t \rightarrow \infty} \varphi_{1}(t ; \cdot, \cdot)=$ $\lim _{c \rightarrow \infty} \varphi_{1}(\cdot ; c, \cdot)=\lim _{\theta \rightarrow \infty} \varphi_{1}(\cdot ; \cdot, \theta)=0$. In the following the dependence of $\varphi_{1}$ on the parameters $c$ and $\theta$ will not be explicitly indicated when unnecessary.

Replacing $u_{\mathrm{eq}}^{\prime}$ in (10)-(11), the solutions $x_{20}(t)$ and $x_{30}(t)$ of the reduced order system are obtained by solving the following system:

$$
\begin{aligned}
\dot{x}_{20}= & -\frac{1}{R_{\mathrm{EPR}} C_{\mathrm{SC}}} x_{20}-\frac{1}{C_{\mathrm{SC}}} \varphi_{1}(t), \\
\dot{x}_{30}= & -\frac{1}{C_{\mathrm{bus}}}\left(\frac{1}{R_{D}}+\frac{1}{R_{H}}\right) x_{30}+\frac{E_{H}}{R_{H} C_{\mathrm{bus}}} \\
& +\frac{1}{C_{\mathrm{bus}}} \varphi_{1}(t) \frac{x_{20}-R_{\mathrm{ESR}} \varphi_{1}(t)-\varphi_{2}(t)}{x_{30}},
\end{aligned}
$$

where $\varphi_{2}=d \varphi_{1}(t ; c, \theta) / d t$. Note that (19) is a stable firstorder linear system with an exponentially decaying input, so its solution is also exponentially decaying. Moreover, the time-varying reduced order system (19) and (20) can be easily transformed into a stationary system embedding the system in $\mathbb{R}^{4}$ and defining the further equations:

$$
\begin{aligned}
& \dot{\xi}_{4}=-c \xi_{4}, \\
& \dot{\xi}_{5}=-\theta \xi_{5}
\end{aligned}
$$

with suitable initial conditions and replacing the exponential terms in $\varphi_{1}$ and $\varphi_{2}$ in (19) and (20). The equilibrium point of (19), (20), and (21) is simply $(0,-\beta / \alpha, 0,0)$, with $\alpha=$ $1 / C_{\text {bus }}\left(1 / R_{D}+1 / R_{H}\right)$ and $\beta=E_{H} /\left(R_{H} C_{\text {bus }}\right)$; thus, using the coordinate change

$$
x_{30}=\xi_{30}+\frac{\beta}{\alpha}
$$

we obtain a new system with equilibrium point at the origin, with

$$
\begin{aligned}
\dot{\xi}_{30}= & -\alpha \xi_{30} \\
& +\phi_{1}\left(\xi_{4}, \xi_{5}\right) \frac{x_{20}-R_{\mathrm{ESR}} \phi_{1}\left(\xi_{4}, \xi_{5}\right)-\phi_{2}\left(\xi_{4}, \xi_{5}\right)}{C_{\mathrm{bus}}\left(\xi_{30}+\beta / \alpha\right)}
\end{aligned}
$$

replacing (20). Here $\phi_{1}$ and $\phi_{2}$ are linear combinations of $\xi_{4}$ and $\xi_{5}$ such that $\varphi_{1}(t)=\phi_{1}\left(\xi_{3}(t), \xi_{4}(t)\right)$ and $\varphi_{2}(t)=$ $\phi_{2}\left(\xi_{3}(t), \xi_{4}(t)\right)$.

The stability of such a system is studied as follows. First, note that only the stability of the variable $\xi_{30}$ has to be discussed, since the other variables are trivially shown to be exponentially decaying. Thus, define the Lyapunov function

$$
V=\frac{1}{2} \xi_{30}^{2}
$$

and compute

$$
\begin{aligned}
\dot{V} & \leq-\alpha \xi_{30}^{2}+\psi(t ; c, \theta)\left|\frac{\xi_{30}}{\xi_{30}+\beta / \alpha}\right| \\
& \leq-2 \alpha V+\psi(t ; c, \theta),
\end{aligned}
$$

where $\psi(t ; c, \theta)$ is a term exponentially decaying with its arguments and the last inequality follows from $\mid \xi_{30} /\left(\xi_{30}+\right.$ $\beta / \alpha) \mid \leq 1$ in $\mathscr{D}$. It remains just to prove that it is possible to choose the parameters $c$ and $\theta$ such that the state remains in $\mathscr{D}$. For the sake of simplicity, assume without loss of generality that $c>\theta$. This assumption is obvious from a physical point of view and simply means that the reference current in the supercap should be quicker than the desired current in the generator. Note that $\psi(t ; c, \theta)$ is a positive exponentially decreasing function, with a quadratic dependence on the exponential terms. Since $c>\theta$, we can neglect $c$ and rewrite (25) as

$$
\dot{V} \leq-2 \alpha V+\eta_{2} e^{-2 \theta t}+\eta_{1} e^{-\theta t}
$$

for suitable positive scalars $\eta_{1}, \eta_{2}$. Solving (26), we have

$V(t)$

$$
\leq e^{-2 \alpha t}\left[V(0)+\eta_{2} \frac{1-e^{-2(\theta-\alpha) t}}{2(\theta-\alpha)}+\eta_{1} \frac{1-e^{-(\theta-2 \alpha) t}}{\theta-2 \alpha}\right]
$$

so that

$$
\lim _{\theta \rightarrow \infty} V(t) \leq e^{-2 \alpha t} V(0)
$$

Hence, if the motion starts within $\mathscr{D}$ it is always possible to choose $\theta$ such that the motion remains in $\mathscr{D}$. Thus, the stability of the reduced order system is proved. By applying the Thikonov's theorem on the Infinite Horizon, the theorem is shown. 


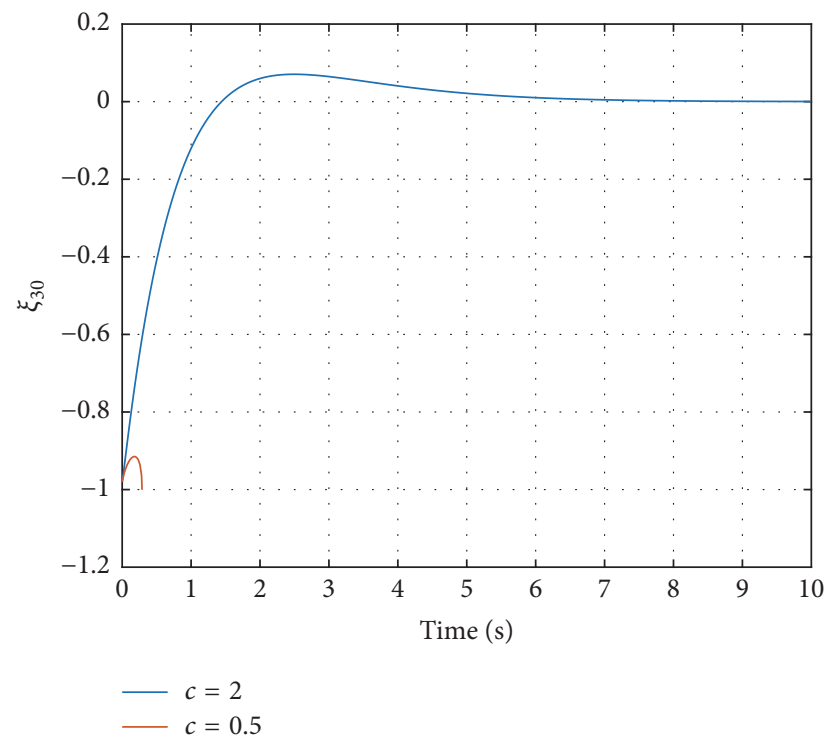

FIGURE 4: Stability of (29) for different values of $c$.

Remark 2. Although the proof is rather long, it is necessary to consider this level of details, since with slow exponential decay the solution of the system may fail to exist, as shown by the following counterexample. Consider the simple case such that the (23) is

$$
\begin{aligned}
\dot{\xi}_{30} & =-\xi_{30}+\frac{-e^{-c t}+e^{-\theta t}}{\xi_{30}+1}, \\
\xi_{30}(0) & =-0.98
\end{aligned}
$$

and assume $\theta=1$. Consider two cases, $c=0.5$ and $c=1$. In the first case (slow exponential term) the solution reaches $\xi_{30}=-1$ in finite time, and from this time instant fails to exist. The fast exponential solution, on the contrary, exists for all $t \geq 0$. This is apparent in Figure 4 , where the two cases are reported. The theorem becomes essential to understand the evolution of system.

Remark 3. In normal aircraft flight, the load changes several times; hence it is necessary to apply the proposed strategy repeatedly, at each load change. In this case the reference current is changed as follows. Assume that the load changes at time intervals $t_{k}, 1 \leq k \leq N$, for a finite number of commutations $N>0$. Then the load reference can be selected as

$$
\bar{y}(t)=\sum_{k=1}^{N} \frac{e^{-\theta\left(t-t_{k}\right)} 1\left(t-t_{k}\right) x_{3}\left(t_{k}\right) \Delta I_{\mathrm{ESD}}\left(t_{k}\right)}{V_{\mathrm{SC}}\left(t_{k}\right)},
$$

where $1(t)$ is the step function, 1 if $t \geq 0$ and 0 otherwise. The control law proposed so far can be used also in this case, provided that a minimum dwell-time condition [18] is satisfied by the loads; that is, two consecutive load changes cannot happen before a minimum time, depending on the value of $\theta$. This condition is very reasonable from a physical point of view and standard in the control of switching systems [19] and can be satisfied by choosing $\theta$ large enough. Note that if many and frequent loads changes have to be considered, complex mathematical tools for the stability of switching have to be considered [20-24].

Remark 4. The main limitation of the above control law is that in order to define the reference function $\bar{y}(t)$ it is necessary to know in advance the changes in the load current (i.e., we need a feedforward component in the control). If a feedforward action is not available, the most we can do is to limit the effect of the load change on the generator. Indeed, since we cannot foresee the load change, only an indirect estimate of load can be obtained after the change has happened, based on a feedback measurement. This means that we must tolerate some quick dynamics in the generator current. The most we can do is to limit the effect of the load change on the generator after a quick transient. Just after a sudden change of load, the controller for the DC/DC converter is maintaining the current $I_{\mathrm{ESD}}$ to its pervious regulation value, and also the bus voltage is kept constant by the capacitor $C_{\text {bus }}$; hence we have the approximate equality at the load change time instant $t_{k}$, as in Remark 3:

$$
\Delta I_{\text {load }}\left(t_{k}\right) \simeq-\Delta I_{\text {bus }}\left(t_{k}\right) .
$$

From an implementation point of view, the reference current $\bar{y}$ is computed from the bus current by using a linear differentiator to compute $\Delta I_{\text {bus }}$ with exponential decay $\theta$; that is, by using the linear filter with transfer function

$$
G(s)=-k \frac{s \tau}{1+s \tau}
$$

with $\tau=1 / \theta$, the minus sign is due to (31) and $k$ is a positive constant.

Remark 5. The control law defined so far is sent to the nonlinear compensator (8). Moreover note that the true control can only assume values 0 or 1 (switch on or off). In the theory of Variable Structure Systems it is shown [25] that if $u$ resulting from 8 assumes only values within the interval $[0,1]$, then implementing the control law with a switching realization such that the control to the switch $S_{1}$ is on if $u$ is larger than 0.5 and off if it is smaller than 0.5 results in a stable and robust implementation of the control law.

\section{Simulation Results}

The simulation is made on the detailed Simulink scheme in Figures 5 and 6, where Figure 6 shows the controlled generator in the rightmost block in Figure 5. The detailed simulation includes switching elements with parasitic resistances in order to test the controller strategy proposed in Section 3. Moreover, as per Remark 4, only the case of pure feedback is considered. Simulations with a feedforward term, where it is assumed that load current is measurable, have been performed, but they are not shown here for brevity, and obviously yields (slightly) better results.

The data used for the model and controller in the simulator are reported in Tables 1 and 2, respectively. The model used for generator is in Simscape Library in 8.7 release of Simulink with parameters in Table 3. 


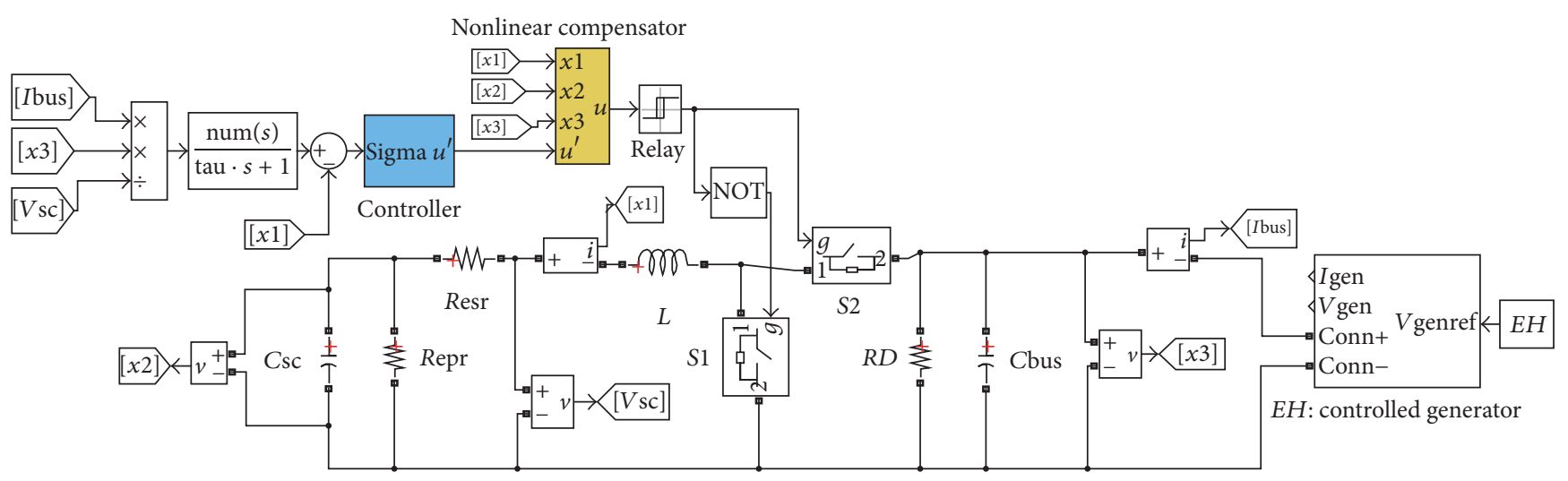

Figure 5: Detailed simulation scheme.

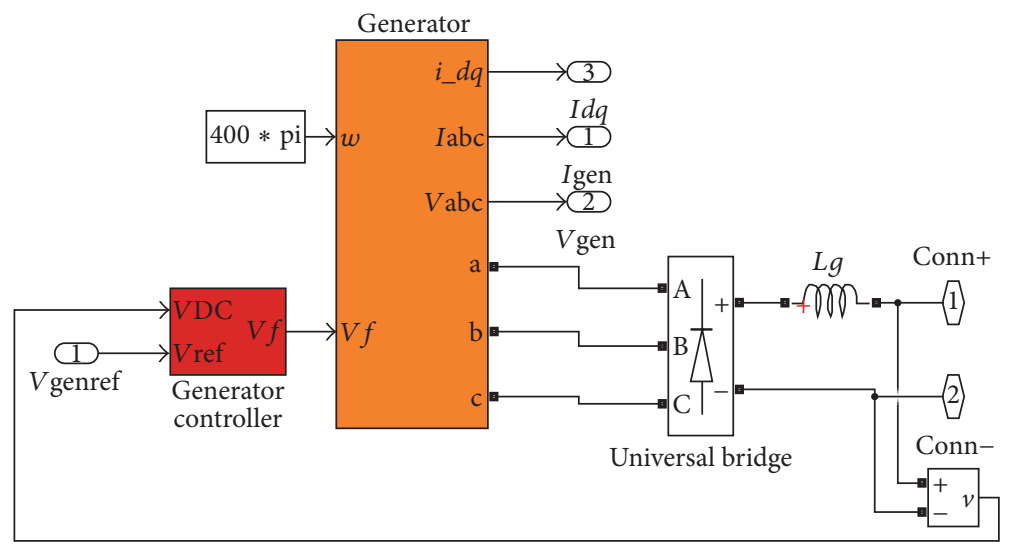

FIGURE 6: $E_{H}$ : controlled generator scheme.

TABLE 1: Model parameters.

\begin{tabular}{lcc}
\hline$C_{\mathrm{SC}}$ & 165 & {$[\mathrm{~F}]$} \\
$R_{\mathrm{EPR}}$ & 10 & {$[\mathrm{k} \Omega]$} \\
$R_{\mathrm{ESR}}$ & 7.5 & {$[\mathrm{~m} \Omega]$} \\
$L$ & 70 & {$[\mathrm{mH}]$} \\
$C_{\text {bus }}$ & 800 & {$[\mu \mathrm{F}]$} \\
$E_{H}$ & 540 & {$[\mathrm{~V}]$} \\
$R_{D 1}$ & 120 & {$[\Omega]$} \\
$R_{D 2}$ & 80 & {$[\Omega]$} \\
$R_{D 3}$ & 30 & {$[\Omega]$} \\
$R_{D 4}$ & 50 & {$[\Omega]$} \\
\hline
\end{tabular}

The detailed scheme is different from the simplified scheme in Figure 2 not only for the presence of switching and parasitic elements, but also because the generator is not simply a battery with the internal resistor. The model of a true generator has been considered, and a simple PI controller has been used to keep the HV side voltage constant. This has been done to show the effect of a change of load on the generator, that is, the main motivation of the whole paper.
TABLE 2: Control parameters.

\begin{tabular}{lcc}
\hline$\epsilon$ & & 0.01 \\
$\gamma$ & & 1 \\
$\tau$ & & 0.1 \\
$k$ & & 4 \\
\hline & & \\
& & \\
& TABLE 3: Generator parameters. & \\
\hline Nominal power & 31.3 & {$[\mathrm{kVA}]$} \\
Voltage & 600 & {$[\mathrm{Vrms}]$} \\
Frequency & 400 & {$[\mathrm{~Hz}]$} \\
Pole pairs & 3 & \\
\hline
\end{tabular}

Different scenarios with different loads changing during the simulation are shown in Figure 7, in order to demonstrate the effectiveness of the control method. At the end the load returns to its initial value.

The presented results also highlight the differences between a controlled system equipped with a supercap and one uncontrolled and without supercap. In Figure 8 the current 


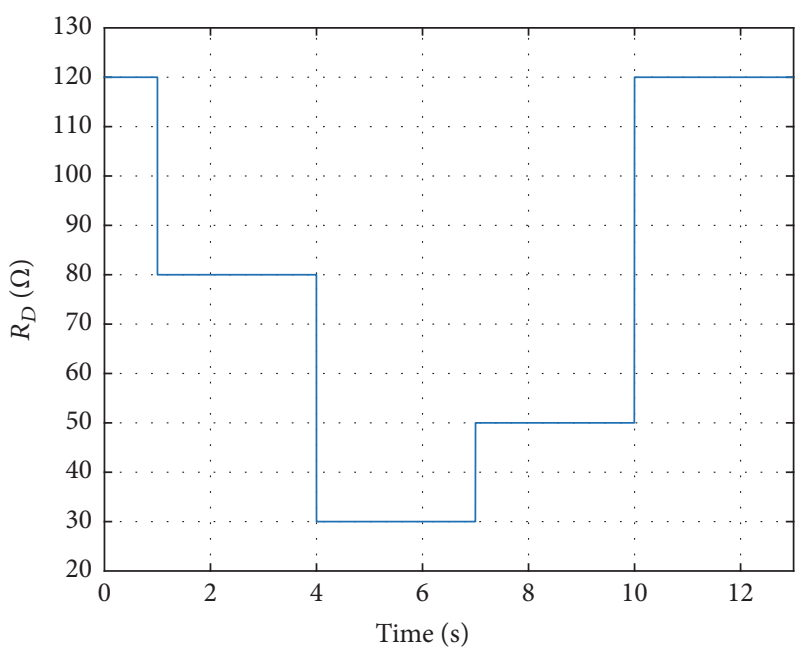

FIgURE 7: Load variation.

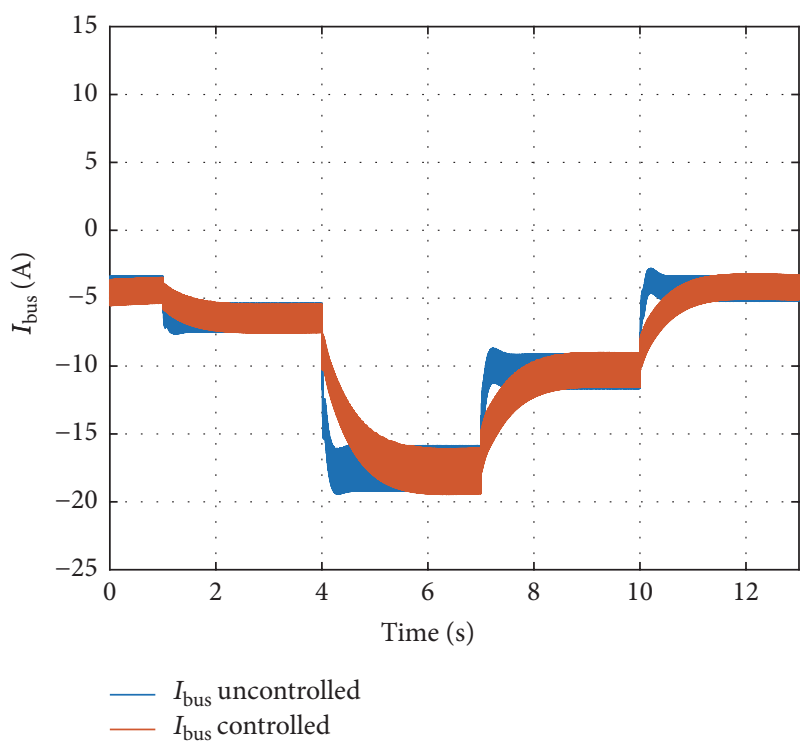

FIGURE 8: HV bus current.

on the main bus supplied by the generator through the rectifier stage is shown and compared with the uncontrolled case. It is clear that the controlled current has a smoother profile. The point here is that the uncontrolled system requires too quick a transient from the generator that in practical implementation, where also rate-limiting requirements have to be considered, can be an undesirable feature. In order to quantify the effect of the control action, let us consider the index

$$
\|\delta u\|_{\left[t_{1}, t_{2}\right]}=\max _{t \in\left[t_{1}, t_{2}\right]}|\dot{u}(t)|
$$

expressing the peak of the derivative of the signal $u$ in a given time interval. Moreover, since in the switching case the use of a derivative would be impractical, a filtering action can be used in combination with the derivative, resulting in a bandpass filter to compute the index (33). Then, we can compare

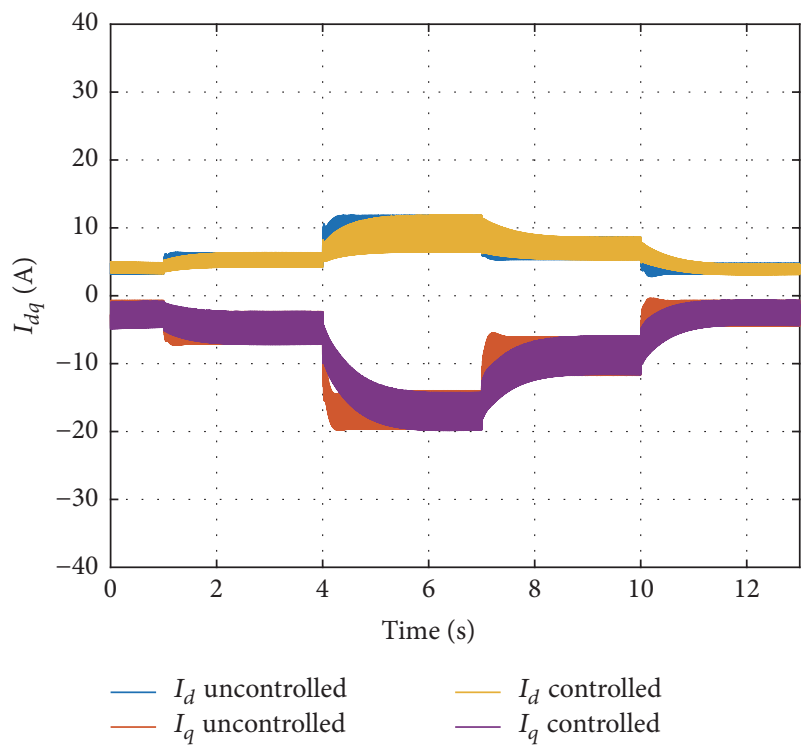

FIGURE 9: Generator $d q$-components.

TABLE 4: Index.

\begin{tabular}{lccc}
\hline$\left[t_{1}, t_{2}\right]$ & Uncontrolled & Controlled & Effectiveness \\
\hline$[0,2]$ & 14.05 & 3.50 & $75.09 \%$ \\
{$[2,5]$} & 57.35 & 18.22 & $68.23 \%$ \\
{$[5,8]$} & 35.77 & 13.84 & $61.31 \%$ \\
{$[8,12]$} & 36.41 & 11.08 & $69.57 \%$ \\
\hline
\end{tabular}

the different values for the controlled and uncontrolled case. The results are shown in Table 4, when using the filter

$$
F(s)=\frac{s}{(0.1 s+1)(0.01 s+1)} .
$$

The fourth column of Table 4 also shows the effectiveness of the control action, that is, the relative improvement due to control $(1-$ controlled/uncontrolled)\%. The beneficial effect on the generator current is shown in Figure 9; the direct and quadrature (in $d q$ Park-Clark transformation) generator current are shown. Also in this case the smoothing effect of the control action is apparent. Obviously, since during a transient depending on the constant $\theta$ the generator supplies a current lower than the one required by the load, the supercap must compensate for the extracurrent required by the load. To show this behaviour, also the supercapacitor current is reported, in Figure 10. In this case some considerations about the supercapacitor current can be drawn, confirming the above goal, that is, to move the stress from generator to the supercap. It is apparent from the sign of the current in the supercap that when the load resistance decreases, the generator current smoothly increases, while the supercapacitor supplies the load with a sudden current peak, and when the load is reduced (e.g., its resistance 


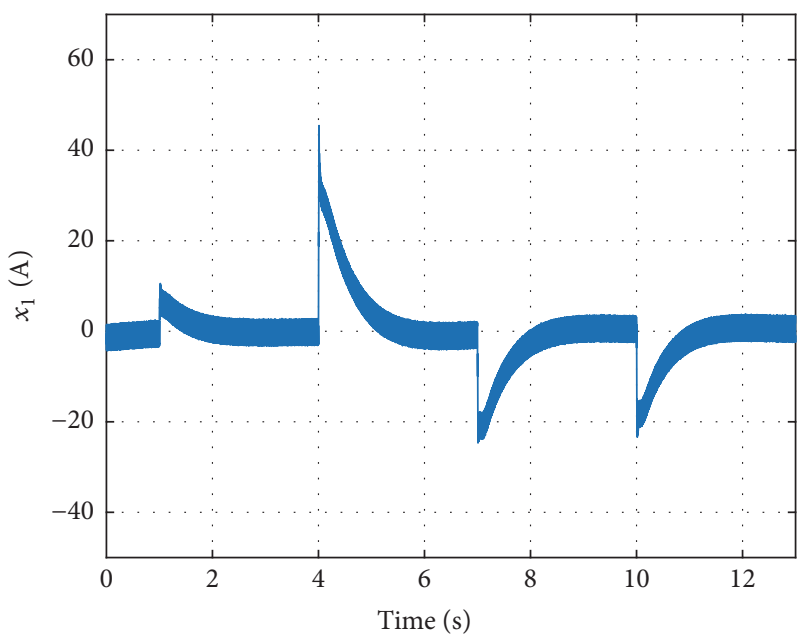

FIGURE 10: Supercapacitor current.

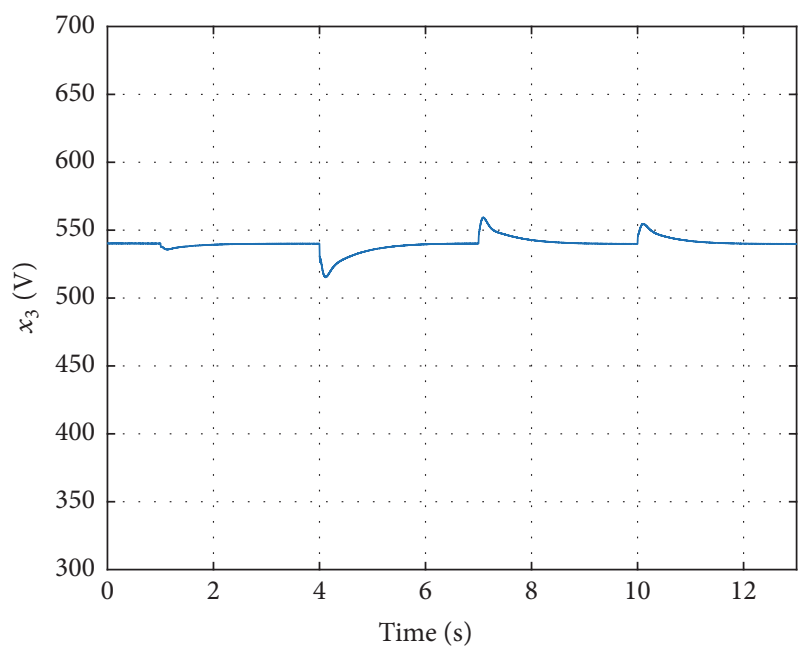

FIGURE 11: Bus voltage.

increases) the supercap draws the extracurrent, allowing the generator to gently reduce its current.

Indeed it is important to remember that the generator is not ideal, so it must be controlled necessary to keep the output voltage close to $E_{H}$. It is the small variation of the voltage on the generator side compared to the voltage on the supercap side that allows the energy to flow from one of the two sources to the other. This is apparent from Figure 11, where the voltage variation on the capacitor $C_{\text {bus }}$ is shown.

Finally, the average control variable is shown in Figure 12. This is the average control after the nonlinear compensator. Note that it only assumes values in the interval $[0,1]$, as required form Remark 5 . The relay in Figure 5 is simply the function

$$
f(x)= \begin{cases}1, & \text { if } x>0.5 \\ 0, & \text { otherwise }\end{cases}
$$

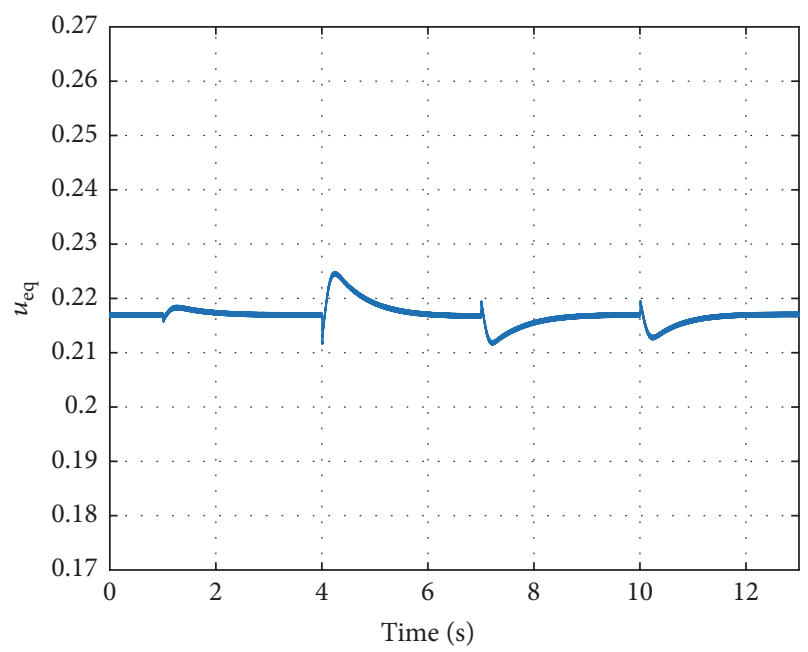

FIGURE 12: Equivalent control $u$.

\section{Conclusions}

In this paper a high-gain control strategy has been presented for controlling the current from and to a supercap, used as a fast energy storage device. The main target of the control is to limit current rushes in the main electric generator, and for this purpose the supercap must supply or withstand fast current peaks. After a nonlinear compensation, a suitable current reference is defined and given to the controller as a profile to be tracked by the supercap current. The tracking problem is solved by resorting to high-gain control, with a formal proof of stability. Moreover, also a realistic switching implementation of the control law is considered by resorting to the theory of variable structure control. Detailed simulations, including switching devices, parasitic elements, and a controlled generator, are provided, and different scenarios are considered, with loads connected and disconnected at random time instants. The results of simulations show the effectiveness of the proposed solution.

\section{Conflicts of Interest}

The authors declare that there are no conflicts of interest regarding the publication of this paper.

\section{References}

[1] C. on Propulsion and E. S. to Reduce Commercial Aviation Carbon Emissions, Commercial Aircraft Propulsion and Energy Systems Research: Reducing Global Carbon Emissions, Space and Aeronautics, National Academies Press, Washington, DC, USA, 2016.

[2] P. Wheeler and S. Bozhko, "The more electric aircraft: Technology and challenges," IEEE Electrification Magazine, vol. 2, no. 4, pp. 6-12, 2014.

[3] D. Schlabe and J. Lienig, "Energy management of aircraft electrical systems-state of the art and further directions," in Proceedings of the Electrical Systems for Aircraft, Railway and Ship Propulsion (ESARS '12), pp. 1-6, October 2012. 
[4] R. Todd, D. Wu, J. A. Dos Santos Girio, M. Poucand, and A. J. Forsyth, "Supercapacitor-based energy management for future aircraft systems," in Proceedings of the 25th Annual IEEE Applied Power Electronics Conference and Exposition (APEC '10), pp. 1306-1312, February 2010.

[5] A. Cavallo, B. Guida, A. Buonanno, and E. Sparaco, "Smart Buck-Boost Converter Unit operations for aeronautical applications," in Proceedings of the 54th IEEE Conference on Decision and Control (CDC '15), pp. 4734-4739, December 2015.

[6] P. Wheeler, A. Trentin, S. Bozhko, and J. Clare, "Regeneration of energy onto an aircraft electrical power system from an electromechanical actuator," in Proceedings of the 2012 Electrical Systems for Aircraft, Railway and Ship Propulsion (ESARS '12), October 2012.

[7] L. Rubino, B. Guida, F. Liccardo, P. Marino, and A. Cavallo, "Buck-boost DC/DC converter for aeronautical applications," in Proceedings of the IEEE International Symposium on Industrial Electronics (ISIE '10), pp. 2690-2695, July 2010.

[8] B. Guida and A. Cavallo, "A Petri net application for energy management in aeronautical networks," in Proceedings of the IEEE 18th International Conference on Emerging Technologies and Factory Automation (ETFA '13), September 2013.

[9] H. Zhang, F. Mollet, C. Saudemont, and B. Robyns, "Experimental validation of energy storage system management strategies for a local DC distribution system of more electric aircraft," IEEE Transactions on Industrial Electronics, vol. 57, no. 12, pp. 3905-3916, 2010.

[10] A. Cavallo, G. De Maria, and P. Nistri, "Robust control design with integral action and limited rate control," Institute of Electrical and Electronics Engineers. Transactions on Automatic Control, vol. 44, no. 8, pp. 1569-1572, 1999.

[11] A. Cavallo and C. Natale, "Output feedback control based on a high-order sliding manifold approach," Institute of Electrical and Electronics Engineers. Transactions on Automatic Control, vol. 48 , no. 3, pp. 469-472, 2003.

[12] D. Wu, R. Todd, and A. J. Forsyth, "Adaptive Rate-Limit Control for Energy Storage Systems," IEEE Transactions on Industrial Electronics, vol. 62, no. 7, pp. 4231-4240, 2015.

[13] A. Cavallo, R. Todd, E. Sparaco et al., "Rate-limiter control comparison for energy storage systems in aerospace applications," in Proceedings of the 8th IET International Conference on Power Electronics, Machines and Drives (PEMD '16), pp. 1-6, Glasgow, UK.

[14] H. Sira-Ramirez and R. Silva-Ortigoza, Control Design Techniques in Power Electronic Devices, Power Systems, Springer, 2006.

[15] D. Wu, R. Todd, and A. J. Forsyth, "Advanced energy management control for energy storage system," in Proceedings of the 7th IET International Conference on Power Electronics, Machines and Drives (PEMD '14), 2014.

[16] H. Khalil, Nonlinear Systems, Prentice Hall, Third Edition edition, 2002.

[17] K.-K. D. Young, P. V. Kokotović, and V. I. Utkin, "A Singular Perturbation Analysis of High-Gain Feedback Systems," IEEE Transactions on Automatic Control, vol. 22, no. 6, pp. 931-938, 1977.

[18] D. Mincarelli, A. Pisano, T. Floquet, and E. Usai, "Uniformly convergent sliding mode-based observation for switched linear systems," International Journal of Robust and Nonlinear Control, vol. 26, no. 7, pp. 1549-1564, 2016.

[19] W. Ni, D. Cheng, and X. Hu, "Minimum dwell time for stability and stabilization of switched linear systems," in Proceedings of the 7th World Congress on Intelligent Control and Automation, WCICA'08, pp. 4103-4108, chn, June 2008.

[20] J. Cortes, "Discontinuous dynamical systems: a tutorial on solutions, nonsmooth analysis, and stability," IEEE Control Systems Magazine, vol. 28, no. 3, pp. 36-73, 2008.

[21] R. Goebel, R. G. Sanfelice, and A. R. Teel, "Hybrid dynamical systems: robust stability and control for systems that combine continuous-time and discrete-time dynamics," IEEE Control Systems Magazine, vol. 29, no. 2, pp. 28-93, 2009.

[22] A. Cavallo, G. Canciello, and B. Guida, "Supervisory control of DC-DC bidirectional converter for advanced aeronautic applications," International Journal of Robust and Nonlinear Control, 2017.

[23] A. Cavallo, G. Canciello, and B. Guida, "Supervised control of buck-boost converters for aeronautical applications," Automatica, vol. 83, pp. 73-80, 2017.

[24] A. Cavallo, G. Canciello, and B. Guida, "Energy storage system control for energy management in advanced aeronautic applications," Mathematical Problems in Engineering, vol. 2017, Article ID 4083132, 9 pages, 2017.

[25] H. Sira-Ramirez, "Sliding motions in bilinear switched networks," Institute of Electrical and Electronics Engineers. Transactions on Circuits and Systems, vol. 34, no. 8, pp. 919-933, 1987. 


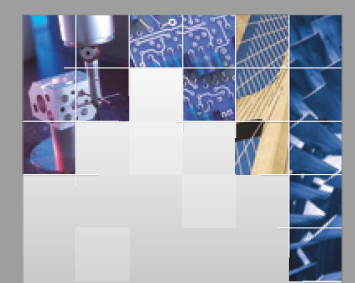

\section{Enfincering}
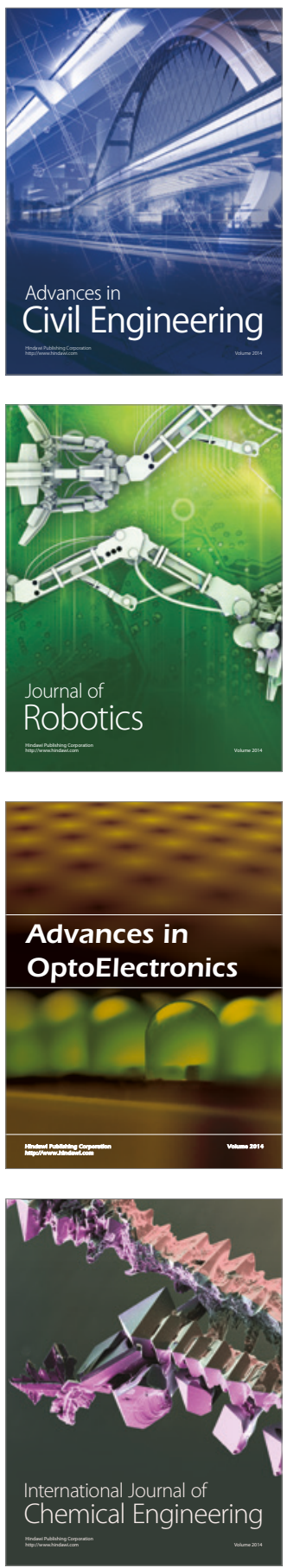

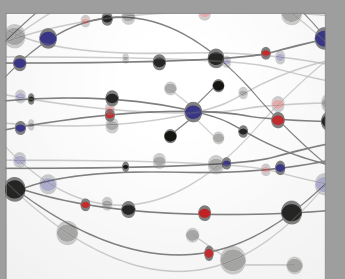

The Scientific World Journal

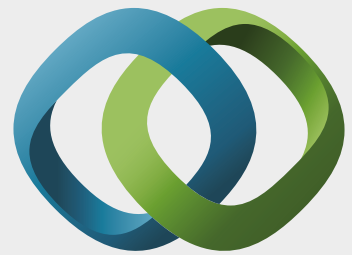

\section{Hindawi}

Submit your manuscripts at

https://www.hindawi.com
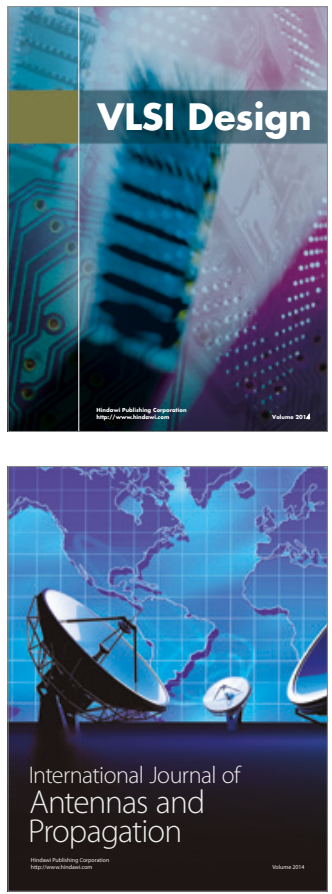

\section{Rotating}

Machinery
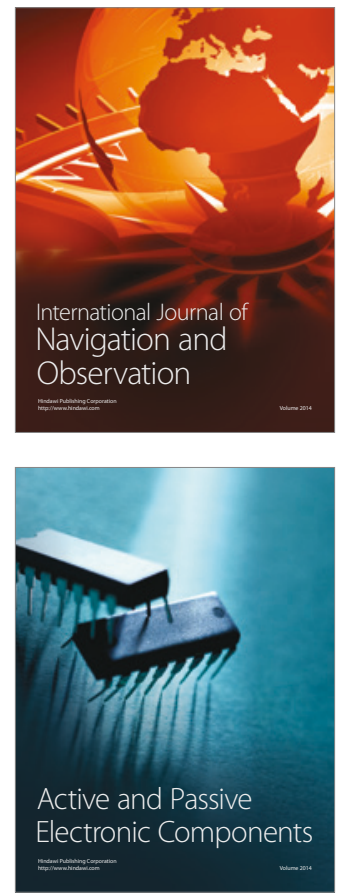
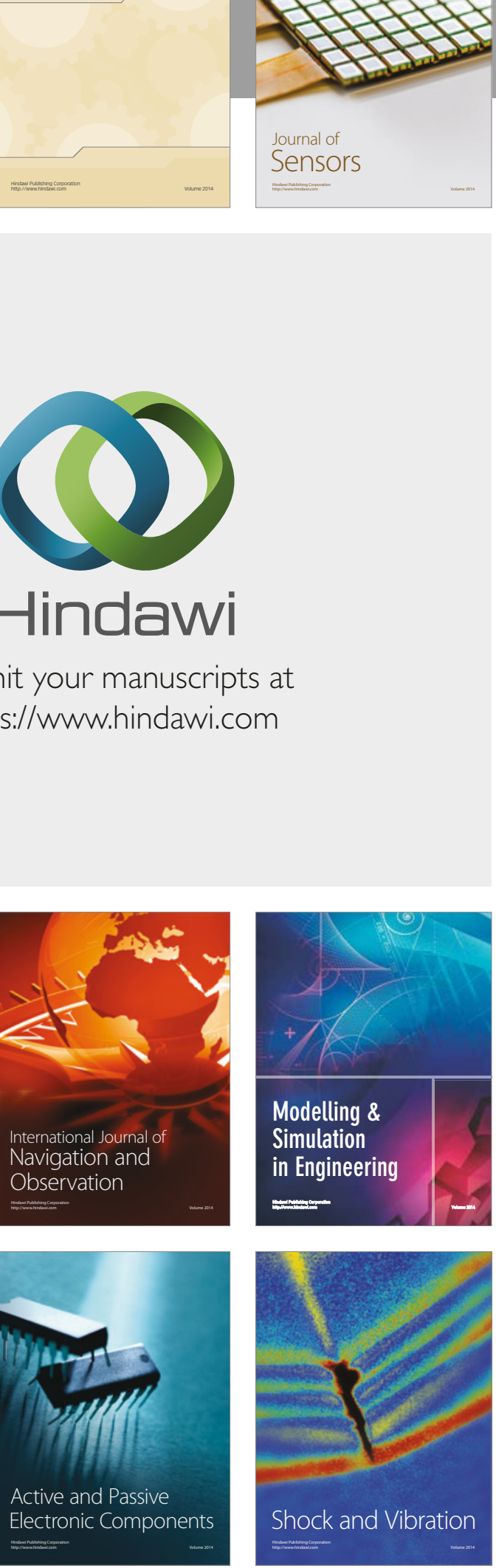
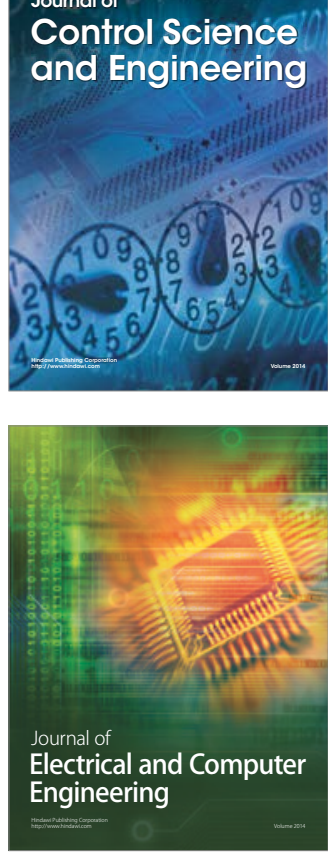

Distributed

Journal of

Control Science

and Engineering
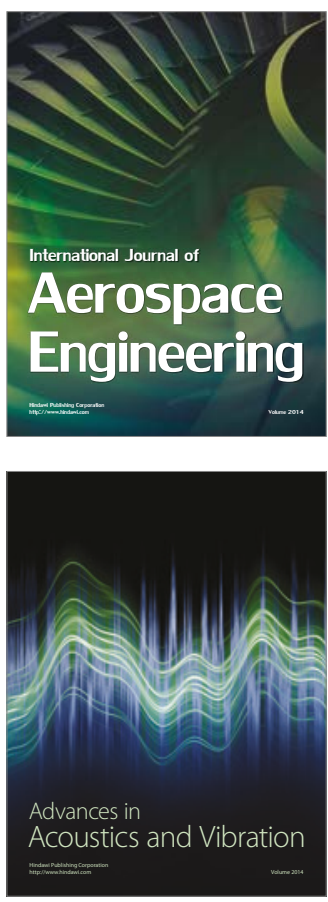

Sensor Networks 